\title{
Consulting with Māori during development of a point-of-care device; translational and experiential findings.
}

Te-Rina J. King-Hudson, PhD student, School of Biological Sciences, University of Canterbury.

Annabel Ahuriri-Driscoll, Lecturer, School of Health Sciences, University of Canterbury.

Renwick C. J. Dobson, Professor of Biochemistry, School of Biological Sciences, University of Canterbury. 


\section{Abstract}

Aim: To consult with Māori on the design and development of a direct-to-consumer point-ofcare (POC) device and gather views on point-of-care testing and biotechnology.

Method: One-on-one interviews and small group hui with self-identified Māori university staff and students $(n=6)$ conducted by an early-career Māori scientist.

Results: Key themes were the importance of achieving improved health outcomes for Māori through addressing known socioeconomic, geographic, and cultural factors that perpetuate health disparities. Other findings were the value of recognising the diversity in modern Māori identities, perspectives, and communities, as well as views on using synthetic biomolecules in medical devices and perceptions of biotechnology, and the potential for cultural overengagement or misplaced focus in consultation.

Conclusion: Direct-to-consumer POC testing may promote kaupapa Māori values such as tino rangatiratanga, whakawhanaungatanga and tikanga, which may help Māori overcome barriers to health care and testing, a key step in achieving improved health outcomes. We describe our approach and experience of consultation as laboratory-based researchers to encourage other researchers/developers to engage directly with Māori during the design process, and report Māori views of synthetic molecules in medical or industrial applications for the first time. 


\section{Introduction}

Significant health disparities exist for Māori in Aotearoa New Zealand due to the ongoing effects of historic colonialism (Jansen et al., 2009; Ellison-Loschmann and Pearce, 2006; Jones et al., 2006; Smith, 1999; Cram et al., 2004). These disparities are amplified for the 16\% of the Māori population living in rural areas (Meredith, 2015), who face additional socioeconomic and geographical barriers to healthcare and diagnostic testing (Robson et al., 2010; Ministry of Health Manatū Hauora, 2012). Point-of-care (POC) testing, the analysis of clinical samples outside the traditional laboratory setting, has emerged as a promising means of addressing this inequity by improving access to testing (Shephard et al., 2005; Blattner et al., 2019). However, currently approved point-of-care testing is only available through hospitals, pharmacies, mobile nurses, or community based assessment centres; imposing increased workloads and pressure to up-skill on healthcare practitioners without addressing the underlying factors to access (Blattner et al., 2010). Innovative approaches such as directto-consumer POC testing (Ayala-Lopez and Nichols, 2020) that may increase accessibility of diagnostic testing for Māori and other historically marginalised groups (Port, 2009) are a key step in improving inequities and health outcomes (Brewer et al., 2019).

We are part of a research team developing a direct-to-consumer POC test or 'screening device' that can detect the presence of a chosen molecule (called markers) in fluids, for applications from screening for health conditions in blood to screening water quality. Our screening device is based on innovative biotechnology (synthetic proteins) that does not use electricity or batteries, is simple to use and understand, can be produced inexpensively, and can be safely shipped. We believe these qualities may be particularly beneficial for rural Māori communities so wanted to find out how consistent our design was with Māori views and outcomes. To this end, we consulted with Māori staff and students at our university on our design and the wider context of diagnostic testing and biotechnology, with detecting diabetes-associated markers in blood as a case study. The purpose of this article is to share our direct, translatable findings and to share our unique perspective as Māori and non-Māori laboratory-based scientists engaging in consultation. 


\section{Methods}

Our approach was adapted from the Kaupapa Māori methodology and methods developed in the Constructive Conversations: Kōrero Whakaaetanga research project (Taupo, 2006; Hipkins, 2004); we interpreted this to mean our research should benefit Māori realities and be directed by Māori, before discussion with the larger research team (Cram et al., 2004; Smith, 1999). A unique aspect of this research was all communication/interviews were carried out by a Māori postgraduate science student with guidance from Māori and non-Māori researchers. For recruitment, a mass email was sent out to Māori UC staff and students by the university's Māori Development Team. Interviewees $(n=6)$ opted in by email and attended a small group (4), or one-on-one (2) hui held on the university campus. Interviews were carried out over a three-week period in March 2020, prior to the initial COVID-19 lockdown, and ranged from 45 mins to 2 hours long with kai, drinks, and koha provided.

Participants comprised urban-based self-identified Māori at various levels of academia; four undergraduate/postgraduate students in social sciences (from 20 - 50 years old), one undergraduate student in engineering (20 years old) and one lecturer of science (over 60 years old). Engagement with te ao Māori varied, with older participants and academics in social science reporting more personal and professional involvement. Participants from social sciences also drew on a combination of personal, whānau, and professional experiences in their responses, presenting a broader range of views. Though participants were from a range of backgrounds, iwi, and locations across Aotearoa (including rural areas in both islands) we acknowledge that there is likely bias given the shared urban/academic background and small sample size.

The interview schedule included questions on designing direct-to-consumer POC testing devices consistent with Māori values, barriers to care and diagnostic testing, and knowledge, perceptions, and views on biotechnology. Interviews were semi-structured with questions displayed on a slide show presentation and asked verbally before open discussion, with responses digitally recorded and later transcribed. The scope of this study was narrow, as it was relatively specific to our device and only university students or staff were able to be recruited and interviewed within the study timeframe due to concerns and limitations of community gatherings amidst the COVID-19 pandemic. 
This research was approved by the University of Canterbury's Human Ethics Committee (HEC 2020/05) and written informed consent was obtained for all participants. 


\section{Results}

Findings from our discussions with Māori academics are presented in a four-tier inverted pyramid of goals, ordered in relative importance to our screening device design from $1-4$ (Figure 1). To achieve the broad primary goal of improving Māori health outcomes with our screening device, objectives (left) from more specific goals lower on the pyramid should be fulfilled as much as possible, without compromising higher-order goals. For example, using more sustainable but expensive materials (Goal 4) would increase the production cost and may increase the cost to the end-user; since cost is a key identified barrier to testing (Goal 2) a suitable option may be using the most cost-effective materials possible while still being environmentally conscious (i.e. product stewardship or alternative recycling schemes). This framework will inform decision-making in the early development stage of our screening device and will be used and evolve alongside on-going consultation with communities.

Figure 1. Decision-making framework ranking the goals and objectives when designing a POC testing device for Māori.

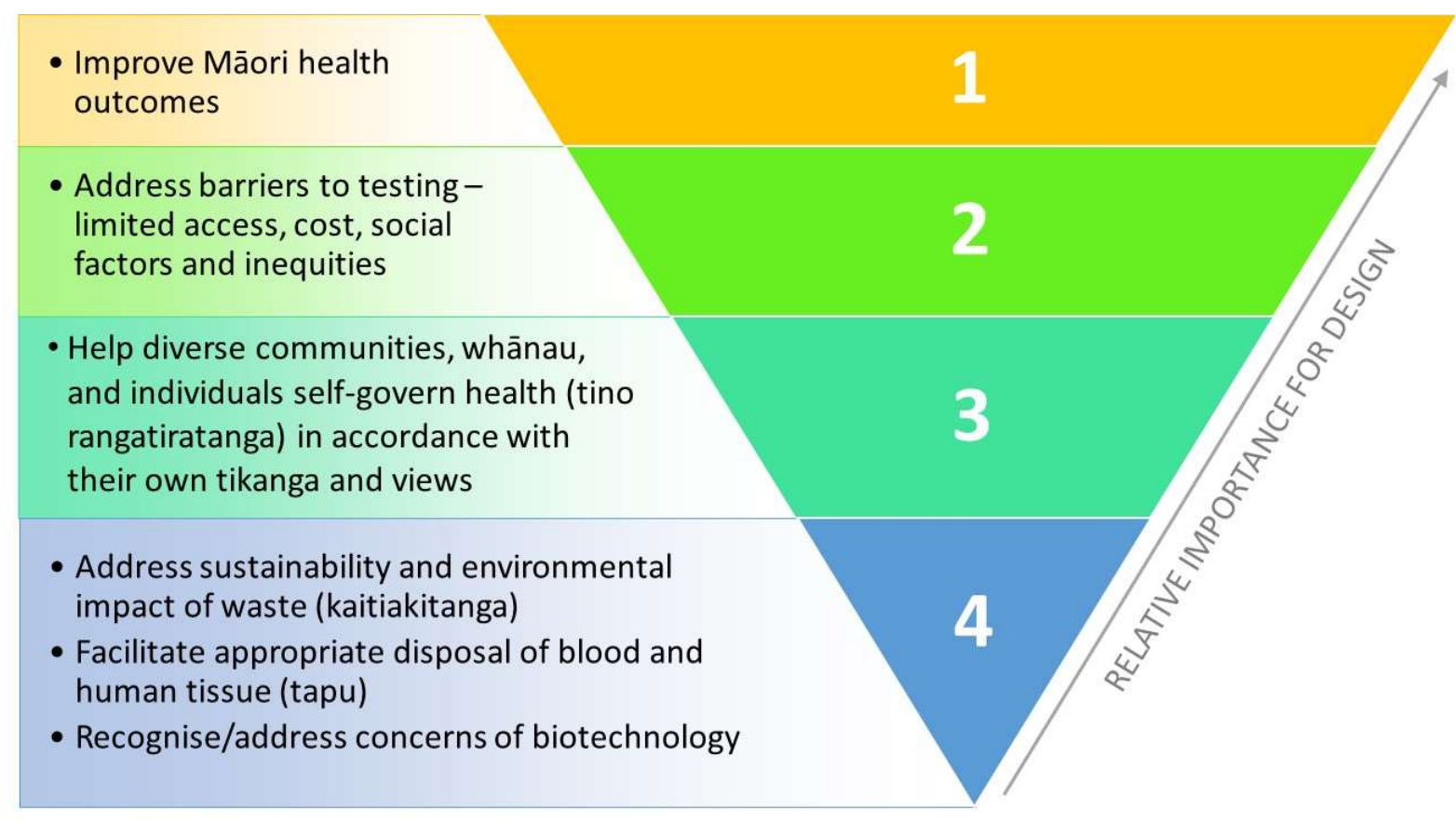


Unexpectedly, factors relating to kaupapa values were ranked as lowest importance in the context of our design. This was a very unexpected finding as we presumed these factors, such as the taking and usage of blood (relating to tapu), protocol around testing and disposal of blood (tikanga), and the environmental impact of a disposable product (kaitiakianga), would present the biggest barriers to use and should be the primary focus of consultation. Instead, most participants believed the design itself should not be the focus, but that the broader social, community, and cultural aspects should be emphasised and empowered.

"I think taking blood and disposal, that's the only big Māori thing involved. Everything else is the social component, the whakawhanaungatanga, manaaki, tino rangatiratanga, it's all of that, which has got nothing to do with the design." - Postgraduate student, social science.

We understand this as the values are not diminished in importance, but that their effect and relevance depends on other higher-order goals or considerations, i.e. views on the tapu and tikanga of blood vary widely across te ao Māori, so creating a simple-to-use design that enables individuals to test in accordance with their own tikanga is of the greatest value and importance. Participants also emphasised that ongoing consultation would be essential to gather conflicting views to address potential concerns, but suggested ways to help address these factors with the design and distribution of the device.

\section{Goal 4: Address cultural concerns of testing, sustainability and biotechnology}

The collection, use, and disposal of blood samples was a key area of discussion, given the tapu around the body and the taking of bodily tissues in te ao Māori (Beaton et al., 2017). Participants reported no personal issues or concerns around the taking of blood in a health context and believed it has become increasingly accepted and normalised, especially with culturally considerate disposal options becoming more available.

"I think things are changing now and becoming more modern. I think people will be surprised when even kaumātua, they're more on board with things because health is important ..." Postgraduate student, social science.

Diagnostic testing may be more acceptable than other contentious applications of genetic or bodily materials like biobanking (Beaton et al., 2017; Hudson et al., 2016) and forensic DNA analysis (Ahuriri-Driscoll et al., 2020), as the kaupapa (purpose) is more in accordance with the positive orientation of whakapapa, i.e. the intent and aim is for users to monitor 
themselves to improve health and wellbeing, rather than having samples collected be used as a tool for criminalisation.

"Making sure that for anyone who has a thing around blood or disposal, that they have appropriate blessings ... for tests done in the house they might want an option to return it to a place, like an option of a place to send it to where it's disposed of appropriately... in case I don't know how to do it myself, if I want it disposed appropriately and I don't know how to do karakia ..." - Postgraduate student, social science.

Nevertheless, participants believed it was important to have options available for culturally appropriate disposal, i.e. regionally specific tikanga processes and karakia, and that further discussion with kaumātua would be important to better understand concerns around the topic.

Sustainability was still considered an important factor to use but ranked as lower priority, which was surprising given the importance of kaitiakitanga or environmental guardianship. Participants agreed addressing sustainability by minimising waste, utilising recycling and product stewardship schemes, and optimising biodegradability would increase uptake and engagement.

"I just feel that you'll get a lot more support if you can show its sustainable or recyclable, just from the whole push for sustainability ..." - Undergraduate student, social science.

However, while most participants believed there would be more support for sustainable initiatives, keeping costs low and addressing social barriers to testing was considered a more important factor of use and engagement.

"Is it possible to be cost-effective and reuseable? ... 'Cause if you reuse it then you're gonna have to factor in that cost ... so the end cost would increase ...

... And with its size, landfill wise it's not gonna be enormous, so if you can get a plastic that does eventually degrade over time ..." - Postgraduate student, social science.

We interpret these comments to mean kaitiakitanga is still highly valued, but in the context of health the potential benefits for individuals and communities takes precedence. We expect that kaumātua and mana whenua may place more importance on kaitiakitanga and sustainability than our participants.

A range of views and acceptability have been reported for biotechnology topics such as genetic testing, stem-cell research, future food, and organ transplants (Beaton et al., 2017; 
Hudson et al., 2012; Roberts and Fairweather, 2004; Hudson et al., 2016), but to our knowledge this is the first research regarding Māori views of synthetic molecules (a form of biotechnology) in medical or industrial applications. Participants with prior knowledge/expertise of biotechnology (i.e. those in science and engineering) held more positive dispositions towards biotechnology-based products than the majority of participants, who were largely unfamiliar with the term outside of media and had reservations around the use of biotechnology-based medical products over traditional electronic products. This preference was attributed to the perceived reliability and certainty of electronics having been tested over time, rather than an aversion to biotechnology. After discussion around the uncertainty of any scientific or medical instrument (Ahuriri-Driscoll et al., 2020) and what biotechnology refers to, most were willing to trial a biotechnology-based medical device.

However, several participants believed that some Māori, particularly older, more traditional and rural communities may still be wary of anything related to biotechnology. This concern may be especially relevant when blood or genetic material is involved (Roberts and Fairweather, 2004; Port, 2009) due to beliefs and restrictions around the body and concerns around the collection and misuse of genetic material.

"Some people are really worried about that [unconsented use or sequencing of genetic material] ... especially I think, coming around back to that word 'biotechnology', that can be freaky for some people.” - PhD student, social science.

Generally, participants favoured keeping terms simple (with more detailed explanations readily available), avoiding overt use of the term 'biotechnology' and jargon such as 'assay', 'diagnostics' and even 'technology'. They believed these terms could be confusing or offputting due to the negative associations with similar but distinct topics, but ultimately believed that the use of biotechnology in this context was acceptable.

\section{Goal 3: Facilitate diverse tikanga and tino rangatiratanga}

Many participants highlighted the benefits of working with marae and communities (Cram et al., 2003) as part of a larger support network, as they can help provide necessary education around using the screening device, interpreting results, and follow-up steps, and the whakawhanaungatanga and support may encourage reluctant individuals to get tested. 
"To be intergenerational, you would have to have someone to explain what [the screening device] was. And if [the result] was positive then what they would have to do about that, 'cause they may not want to [follow up], so you would have to have the whole whakapapa network. So if it was marae based there's support there ...

....and if it's coming from [marae] they may be able to sway people to try it ... and then they can also help with the disposal in a culturally competent setting." - Undergraduate student, social science.

Working with communities directly will allow users to use the test in a way that is consistent with their own tikanga (i.e. karakia upon collection and disposal, burning, keeping, or burying their used device) as communities have different tikanga, unique restrictions and considerations.

“'Cause each [community] has a different mindset ... I know up North, there's a tribe up there that don't allow, they don't like organ transplants, cause of the whole issue with the body ..." - Undergraduate student, social science

Given the diversity of views, beliefs, and engagement across te ao Māori (Roberts and Fairweather, 2004; Hudson et al., 2012), some participants further recommended working with alternative services like pharmacies (Beyene et al., 2021)) for those not connected to a marae or community networks, as well as providing more modern means of distribution and assistance (i.e. online shopping and video tutorials) for younger or more urban-based individuals.

"... Also in the pharmacies, because they get out and deliver stuff in the rural areas as well, so for people who many not be as connected to their marae so they won't miss out. Just finding all those community channels and networks." - Postgraduate student, social science.

Participants highlighted the importance of tino rangatiratanga or self-determination and believed it was a key advantage of our design.

"'Cause I think in terms of values it's very important for people to feel like they're selfdetermining ... that they're having their own say of what they want to do. ... It's [the community/individuals] doing it, you're not doing something to them ... tino rangatiratanga ... it's about values and relating that back to everything ..." - Postgraduate student, social science.

Enabling users to control the circumstances around their own testing and to be selfdetermining may also help to address implicit concerns regarding the scientific/academic 
nature of our group and device, as the kaupapa is for communities to help and test themselves rather than having researchers take from or act upon them.

\section{Goal 2: Minimise barriers to testing}

Māori, especially older Māori and those living in rural areas experience additional socioeconomic barriers relating to care and testing. Participants discussed several barriers, including feelings of whakamā (culturally appropriate shame or embarrassment) (Cram et al., 2003), limited access to primary-care due to low availability and high 'load' on health services (Ellison-Loschmann and Pearce, 2006; Jansen et al., 2009; Robson et al., 2010), difficulties self-managing chronic and/or co-morbid health conditions confounded by limited access to care, and ongoing effects of historically poor healthcare practitioner/patient relationships (Beyene et al., 2021; Cram et al., 2003) - all of which may be better addressed by POC testing than traditional primary-care based screening.

“... The barrier for the older people not going might be I'm embarrassed to go to the doctor, or it costs too much." - Undergraduate student, social science.

"I know GP practices in my town [rural North Island] are so busy you can't even get in. So if that's taking the initial load off the first appointment, and you only get people coming in when they do detect something that takes a huge load off." - Postgraduate student, social science.

Besides these social barriers, cost was the main concern and barrier to health care and diagnostic testing for participants and their whānau. The idea of 'cost' extended beyond the upfront cost of appointment/testing fees, also including travel costs, appointment and testing fees for other health concerns, taking time off of work and arranging childcare.

"When I spoke to some relations about your project, their first question was "how much does it cost?" - Undergraduate student, social science.

"Screening a whole entire family [at the doctors] ... it's gonna be expensive and so many appointments you've got to make ..." - Postgraduate student, social science.

“... And that could be an added thing on top of other health issues you're already going to the hospital for, you're already going to the doctor all the time for ..." - Postgraduate student, social science. 
A major advantage of our design is avoiding these additional costs of traditional primary health care, but hidden costs associated with implementation and quality assurance are one noted challenge with POC testing (Blattner et al., 2019) that should be accounted for and minimised at later stages of development to ensure the cost to users remains low.

Overall, key identified barriers to accessing health services and testing were broad economic/financial concerns, social and cultural factors relating to primary-care and healthcare practitioners, and the low availability of rural services. Our screening device and other POC tests may help alleviate these barriers, particularly in rural areas, where it may help reduce the 'load' on primary health care and increase efficiency by allowing individuals to access testing directly.

\section{Goal 1: Improve Māori health outcomes}

Foremost, participants emphasised the importance of improving Māori health outcomes. Most believed that although many factors would affect the overall support and engagement from Māori, innovative POC testing with the capacity to improve Māori health outcomes should be produced and distributed to the community as soon as possible.

"The important thing is to get it diagnosed. Even though you might lose out on those things [sustainability, culturally-appropriate disposal services, multi-level support networks] for a while, it's a prototype, you can fill in those gaps, it just seems to be important to get it out there and get people doing it." - Postgraduate student, social science.

Achieving this goal with our design depends on how successful we are in fulfilling the lower order goals discussed. Ideally, we will be able to address or mitigate all goals and concerns, but when this is not possible the framework developed here will help us make compromises consistent with key values and outcome.

\section{Other findings}

Helpfully, some participants outlined a general process for later development stages. They recommended we approach local marae for further consultation and pilot studies of our prototype in the community, which will allow users to give more informed and practical feedback on the design and operation. This could be followed by iterative processes of larger- 
scale implementation and consultation until an optimised product is reached. An added benefit of this approach is that those communities who may benefit more from the product would be meaningfully engaged and involved in the development process, which may increase uptake and use of the final product.

Some participants warned there was a potential risk of 'over-engaging' with the cultural considerations of design, which may slow down or detract from the larger focus of improving Māori health. They believed that besides the use of blood and disposal of the device, the design itself was less important to consider and consult upon than the social implications and determinants of its use and outcome (at least in the earlier stages of design and development).

"I think there's a huge over-emphasis on how culturally engaged people are ... like me, I'd rather do a test and I don't think twice about how my blood's being disposed, and a lot of people I know are like that ... if I ask them they're like 'who cares as long as I'm alive and I'm healthy, I don't care' ...” - Postgraduate student, social science.

Another key point was the benefits of having a Māori researcher lead discussion, as the shared ancestry and cultural understanding (Hudson et al., 2012) made it easier for participants and researcher to navigate the interface of biomolecular research/biomedical technology and socioeconomic realities for Māori. Some participants believed having Māori researchers lead consultation would likely improve engagement with communities that may otherwise be wary or mistrusting of scientists (Wilcox et al., 2008).

"Someone like you, going back to your iwi and talking about [this project], they'll go "oh she's a nice girl, we'll do this because we like her and what she's about', but for [non-Māori] it would be quite different ..." - Lecturer of science.

Increasing our understanding of te ao Māori principles and realities as researchers/developers will facilitate more productive communication during consultation, towards a more relevant design capable of improving health outcomes. Therefore, increasing dialogue between researchers and developers benefits researcher, research, and most importantly Māori communities. 


\section{Discussion and Reflection}

Perhaps unsurprisingly our findings reflect the general principles of Kaupapa Māori (Smith, 1999), with the key expected outcome of developing our screening device being the improvement of health and social realities for Māori (Eketone, 2008). Other significant outcomes relate to values of tino rangatiratanga and tikanga, highlighting the importance of respecting Māori autonomy by working alongside Māori and of recognising and legitimising Māori beliefs, practices, and ways of doing things throughout the development and implementation process. This requires consideration of the diversity within te ao Māori, including differences between individual regions, iwi, hapū, and communities, as well as the diverse range of knowledge and te ao Māori engagement of modern Māori identities. Again, this is best achieved by working with Māori communities or providing tools and opportunities for Māori to help themselves, ideally with Māori researchers at the interface between larger research teams and Māori communities (Wilcox et al., 2008; Ahuriri-Driscoll et al., 2007). Interestingly, outcomes around values such as kaitiakitanga and tapu, while still considered highly important, were deemed less influential than addressing socioeconomic barriers in achieving improved health realities.

What was surprising was the relative indifference in this cohort for how hui were held, with some participants preferring to defer karakia and formalities of proceedings. Adapting and responding to diversity by asking participants their preferred kawa (process/procedure) rather than enforcing a strict process may better align with the intentions of tikanga principles (Hipkins, 2004) and links to the idea of cultural 'over engagement' raised by some participants. This may only apply for those used to working/studying within a Western institution (i.e. university) as holding hui in marae or other settings would be a very different situation; generally, we believe it is important to find a balance between adherence to protocols and adaptation for genuine and meaningful engagement.

Recruiting participants was the most challenging and time-consuming part of the consultation process, as initial attempts to recruit Māori staff were unproductive until we engaged the university's Māori Development Team through our department's kaiārahi. Many potential connections emerged from hui, with participants offering further networking opportunities with local marae and rūnanga. This speaks to the practical value of whakawhanaungatanga, making culturally meaningful relationships, as an essential and ongoing part of the consultation process. However, we have learnt that this may pose an initial barrier for 
laboratory-based scientists without existing connections, who we encourage to first seek guidance from Māori intermediaries or cultural advisors (Wilcox et al., 2008).

Lastly, participants agreed that having a Māori scientist direct research and consultation facilitated discussion around the interface of Western scientific knowledge and te ao Māori realities and worldviews, as researcher and participants recognised the validity and limitations of each paradigm in different situations (Hudson et al., 2012). Given the current underrepresentation of Māori scientists, especially within biochemical and molecular sciences, (McAllister et al., 2020) this is a disadvantage in the short-term and further cause to actively encourage, support, and uplift aspiring Māori scientists, to help facilitate more productive and meaningful engagement between Māori and Western science and development. 


\section{Conclusion}

Here, we have outlined our approach and experience of consulting with Māori academics on the development of a novel diagnostic testing device. Our consultation was based on Kaupapa Māori methodology/practice, with a Māori scientist leading discussion and interpretation. Our key finding was that the most positively impactful goal/outcome of developing innovative testing biotechnology is to improve Māori health outcomes. This can be achieved by addressing identified socioeconomic barriers, enacting key values including tino rangatiratanga, whakawhanaungatanga, and tikanga, and considering the diverse Māori cultural practices, communities and identities in Aotearoa New Zealand. Other interesting findings include the relative acceptability of biotechnology in medical applications, the potential risk of cultural 'over-engagement' and the benefits of having Māori scientists to help navigate the interface of Western research/development and Māori realities. Whilst the scope of consultation in the present study was limited, we intend to continue consultation at later stages of development and encourage other scientists and innovators to meaningfully integrate consultation into their design and development. 


\section{Acknowledgements}

We would firstly like to acknowledge and thank our participants for their valuable time, suggestions, and willingness to share their knowledge and insights. We would also like to thank Dr. Abby Suszko and the University of Canterbury Māori Development Team for their advice, guidance, and for kindly sharing their resources.

\section{Funding}

Funding was provided by MBIE Smart Idea’s Grant UOCX1706. 


\section{References}

Ahuriri-Driscoll A, Hudson M, Foote J, et al. (2007) Scientific Collaborative Research with Māori Communities: Kaupapa or Kūpapa Māori? AlterNative: An International Journal of Indigenous Peoples 3: 60-81.

Ahuriri-Driscoll A, Tauri J and Veth J. (2020) Māori views of forensic DNA evidence: an instrument of justice or criminalizing technology? New Genetics and Society: 1-18.

Ayala-Lopez N and Nichols JH. (2020) Benefits and Risks of Direct-to-Consumer Testing. Archives of Pathology \& Laboratory Medicine 144: 1193-1198.

Beaton A, Hudson M, Milne M, et al. (2017) Engaging Māori in biobanking and genomic research: a model for biobanks to guide culturally informed governance, operational, and community engagement activities. Genetics in Medicine 19: 345-351.

Beyene K, Chan AHY, Bandreddi NST, et al. (2021) Patient satisfaction with community pharmacist-led anticoagulation management services and its relationship with patient characteristics in New Zealand. International Journal of Clinical Pharmacy 43: 154164.

Blattner K, Beazley CJ, Nixon G, et al. (2019) The impact of the introduction of a point-ofcare haematology analyser in a New Zealand rural hospital with no onsite laboratory. Rural Remote Health 19: 4934.

Blattner K, Nixon G, Jaye C, et al. (2010) Introducing point-of-care testing into a rural hospital setting: thematic analysis of interviews with providers. Journal of Primary Health Care 2: 54-60.

Brewer N, Foliaki S, Bromhead C, et al. (2019) Acceptability of human papillomavirus selfsampling for cervical-cancer screening in under-screened Māori and Pasifika women: a pilot study. The New Zealand medical journal 132: 21-31.

Cram F, Phillips H, Tipene-Matua B, et al. (2004) A 'Parallel process'? beginning a constructive conversation about a Mäori methodology. Journal of bioethical inquiry 1: 14-19.

Cram F, Smith L and Johnstone W. (2003) Mapping the themes of Maori talk about health. The New Zealand medical journal 116.

Eketone A. (2008) Theoretical underpinnings of Kaupapa Maori directed practice. MAI Review.

Ellison-Loschmann L and Pearce N. (2006) Improving access to health care among New Zealand's Maori population. American journal of public health 96: 612-617.

Hipkins R. (2004) Methodological Overview: The development and implementation of the Constructive Conversations/ Korero Whakaaetanga contact group methodology. Christchurch: Social Science Research Centre: University of Canterbury.

Hudson M, Roberts MR, Smith LT, et al. (2012) The art of dialogue with indigenous communities in the new biotechnology world. New Genetics and Society online: 1124.

Hudson M, Southey K, Uerata L, et al. (2016) Key informant views on biobanking and genomic research with Māori. The New Zealand medical journal 129: 29-42.

Jansen P, Bacal K and Crengle S. (2009) He Ritenga Whakaaro: Māori experiences of health services. . Auckland: Mauri Ora Associates.

Jones R, Crengle S and McCreanor T. (2006) How tikanga guides and protects the research process: insights From the hauora tāne project. Social Policy Journal of New Zealand.

McAllister TG, Naepi S, Wilson E, et al. (2020) Under-represented and overlooked: Māori and Pasifika scientists in Aotearoa New Zealand's universities and crown-research institutes. Journal of the Royal Society of New Zealand: 1-16. 
Meredith P. (2015) Urban Māori. Available at: http://www.TeAra.govt.nz/en/urbanmaori/page-1;\%20and\%20Statistics\%20New\%20Zealand.

Ministry of Health Manatū Hauora. (2012) Mātātuhi Tuawhenua: Health of Rūral Māori 2012. Wellington: Ministry of Health.

Port W. (2009) Māori culture and genetic technology. MAI Review.

Roberts M and Fairweather J. (2004) South Island Maori perceptions of biotechnology.

Robson B, Purdie G and Cormack D. (2010) Unequal Impact II: Māori and Non-Māori Cancer Statistics by Deprivation and Rural-Urban Status, 2002-2006. Wellington: Ministry of Health.

Shephard MD, Mazzachi BC, Shephard AK, et al. (2005) The impact of point of care testing on diabetes services along Victoria's Mallee Track: results of a community-based diabetes risk assessment and management program. Rural Remote Health 5: 371.

Smith LT. (1999) Decolonizing methodologies : research and indigenous peoples: Zed Books.

Taupo K. (2006) Close Encounters of the Genetic Testing Kind: Negotiating the interfaces between Matauranga $\mathrm{N}$ ori and other knowledge systems.

Wilcox PL, Charity JA, Roberts MR, et al. (2008) A values - based process for cross cultural dialogue between scientists and Māori. Journal of the Royal Society of New Zealand 38: 215-227. 